OPEN ACCESS

Edited and reviewed by:

Alessandro Passardi,

Romagnolo Scientific Institute for the

Study and Treatment of Tumors

(IRCCS), Italy

*Correspondence:

Xinyu Shao

xinxinyuxin0711@163.com

Yizhou Yao

yzyao@suda.edu.cn

${ }^{\text {t}}$ These authors have contributed equally to this work

Specialty section: This article was submitted to Gastrointestinal Cancers: Colorectal Cancer,

a section of the journal

Frontiers in Oncology

Received: 10 October 2021 Accepted: 11 October 2021 Published: 25 October 2021

Citation:

Duan T, Zhou D, Yao Y and Shao X (2021) Corrigendum: The Association of Aberrant Expression of FGF1 and mTOR-S6K1 in Colorectal Cancer.

Front. Oncol. 11:792453.

doi: 10.3389/fonc. 2021.792453

\section{Corrigendum: The Association of Aberrant Expression of FGF1 and mTOR-S6K1 in Colorectal Cancer}

\author{
Tinghui Duan ${ }^{1 \dagger}$, Diyuan Zhou ${ }^{2 \dagger}$, Yizhou Yao ${ }^{2 *}$ and Xinyu Shao ${ }^{3 *}$ \\ ${ }^{1}$ Department of Medical Imaging, The Affiliated Guangji Hospital of Soochow University, Suzhou, China, ${ }^{2}$ Department of \\ General Surgery, The First Affiliated Hospital of Soochow University, Suzhou, China, ${ }^{3}$ Department of Gastroenterology, The \\ Affiliated Suzhou Hospital of Nanjing Medical University, Suzhou Municipal Hospital, Gusu School, Nanjing Medical University, \\ Suzhou, China
}

Keywords: colorectal cancer, FGF1, mTOR-S6K1 pathway, prognosis, survival

\section{A Corrigendum on:}

The Association of Aberrant Expression of FGF1 and mTOR-S6K1 in Colorectal Cancer by Duan T, Zhou D, Yao Y and Shao X (2021) Front. Oncol. 11:706838. doi: 10.3389/fonc.2021.706838

In the original article, there was a mistake in Figure $\mathbf{7 B}$ as published. We identified a minor error in image-misusing was made. The corrected Figure 7 appears below.

The authors apologize for this error and state that this does not change the scientific conclusions of the article in any way. The original article has been updated.

Publisher's Note: All claims expressed in this article are solely those of the authors and do not necessarily represent those of their affiliated organizations, or those of the publisher, the editors and the reviewers. Any product that may be evaluated in this article, or claim that may be made by its manufacturer, is not guaranteed or endorsed by the publisher.

Copyright $\odot 2021$ Duan, Zhou, Yao and Shao. This is an open-access article distributed under the terms of the Creative Commons Attribution License (CC BY). The use, distribution or reproduction in other forums is permitted, provided the original author(s) and the copyright owner(s) are credited and that the original publication in this journal is cited, in accordance with accepted academic practice. No use, distribution or reproduction is permitted which does not comply with these terms. 

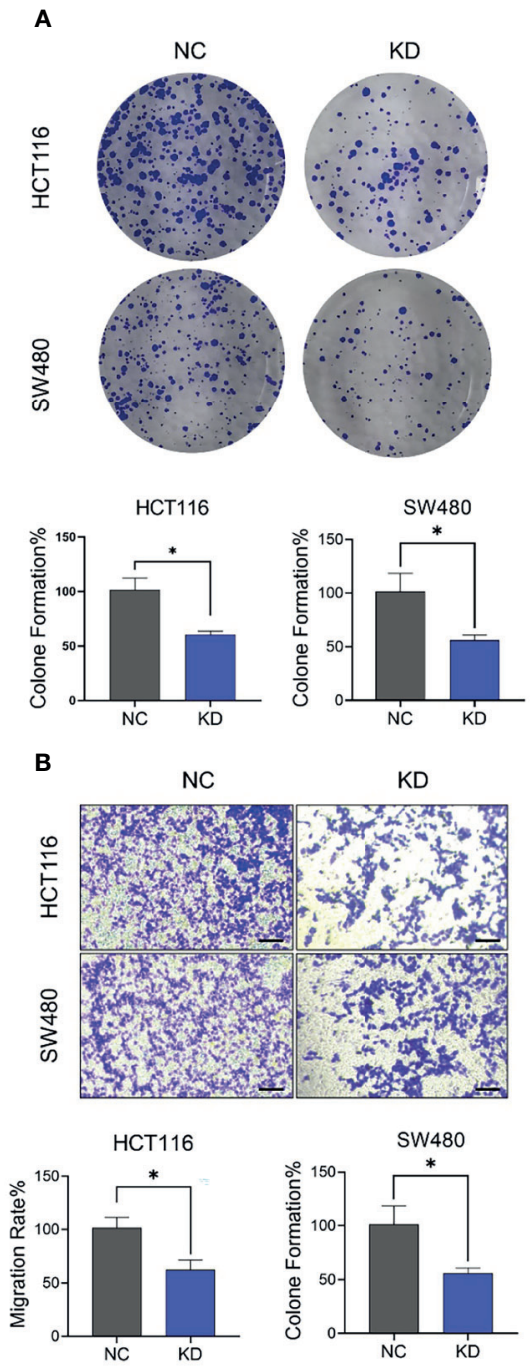

C
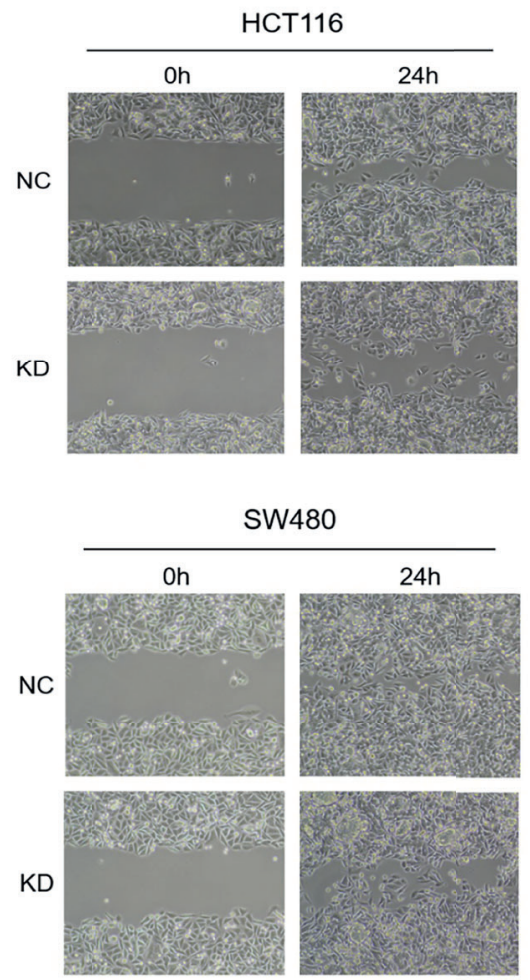

\section{.}
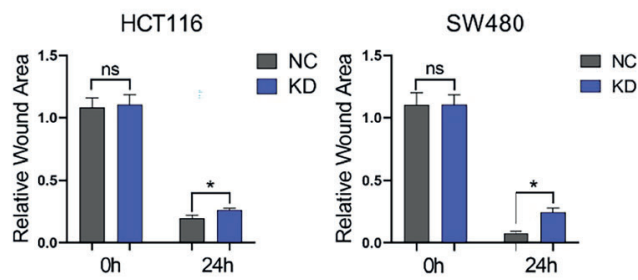

FIGURE 7 | FGF1 promotes proliferation and migration ability of CRC cells. (A, B) Colony formation capacity (A) and migration rates (B) of FGF1-KD CRC cells. CRC, colorectal cancer. (C) Wound healing assays were carried out at $24 \mathrm{~h}$ after transfection in 6-well plates. The gap width was measured using Open Lab software. (D) The wound rate was calculated and displayed graphically according to the measured results by Open Lab software. NC, negative control; KD, FGF1shRNA. Data are presented as mean $\pm \mathrm{SD}(\mathrm{n}=3)$. Ns, no significance, ${ }^{\star} P<0.05$. 